\title{
Psychoanalysis of the Doctrine of Acute Pneumonia
}

\author{
Igor Klepikov, MD*, \\ Professor, retired, USA
}

*Corresponding Author: Igor Klepikov, MD, Professor, retired, USA.

\section{LETTER TO THE EDITOR}

It is difficult to predict how the founder of psychoanalysis, Sigmund Freud, would react to an attempt to link his theory and the method of treating mental disorders based on it (1) with such a purely physical disease as acute pneumonia (AP). It is unlikely that such an innovation could cause full approval and support. However, in this context, we are not talking about psychoanalysis as a therapeutic method for AP. In this case, only the diagnostic features of this technique are of interest. The essence of psychoanalysis, which is based on the search for the causes of the so-called echoes of the past, as well as the connection between conscious and unconscious phenomena, can be useful in order to understand the causes of errors and paradoxes that exist in solving the problem of AP. In other words, we are not talking about the nuances of the disease itself, but about the peculiarities of its nature, since the interpretation of the essence of AP determines the principles of treatment and the final results.

AP is one of the oldest nosologies known to modern medicine. Its history in medicine goes back more than 2,500 years (2). For many centuries, this disease has had relatively constant conditions of occurrence and development, as well as certain traditions of treatment. The lack of fundamental knowledge and the complexity of objective testing did not allow the old medicine to improve the medical care that was selected empirically. The era of antibiotics marked the beginning of not only a new therapeutic direction, but also the biological process of changing the etiological factors of pneumonia. The growing resistance of microorganisms, the constant need to develop new antimicrobial drugs, the frequent change of the leading pathogens of inflammation, and, finally, the increasing role of viruses in the etiology accompanied the entire period of antibacterial therapy of AP.

Along with the biological consequences, prolonged attention to the leading role of antibiotics in treatment has distorted the understanding of the underlying disease and given a negative didactic effect. The current understanding of AP focuses on the action of the pathogen and leaves aside the mechanisms of development and the influence of the focus of inflammation itself. Existing views on the nature of this disease direct the solution of the problem along a narrow etiotropic path and ignore the features of the disease. A brief analysis of the current concept of AP, combined with a number of well-known facts, as well as obvious contradictions between the theoretical and practical sides of this problem, is necessary in order to understand the causes of distortions and misconceptions.

AP throughout its history did not belong to the category of dangerous infections and contact with such patients did not require special antiepidemic and protective measures. The emergence of microbiology allowed us to establish that the pathogens of AP are conditionally pathogenic concomitant microflora of the body. Discovered in the second half of the 19th century, pneumococcus got its name because of the greater frequency of detection in this disease (3). Remaining the leader in this list for many subsequent decades, it was not the only microbial factor in the etiology of AP, so the inflammation was considered as non-specific.

The first experience of using antibiotics laid the foundation for future illusions about this medical care option, the effectiveness and ease of use of which seemed to many a long-term achievement. After prescribing a course of treatment with antibiotics, the doctor actually calmly observed the recovery of patients. However, such a sinecure could not last long enough or permanently.

Antibiotics, unlike drugs of classical pharmacology, do not directly affect the structures and processes of the body itself. They selectively act only on the microflora present. Bacteria, in turn, are themselves biological 
objects and have the ability to adapt and change. These qualities were convincingly demonstrated by representatives of the microbial world throughout the entire period of antibiotic use, which was accompanied by a number of nonstandard phenomena. The growing resistance of microflora to antibiotics required the development and introduction of more and more new drugs. A group of antibiotic-resistant strains that can occur as part of the symbiotic microflora in healthy people appeared and began to grow. Over the past decades, AP has lost the stability of its etiology, which is characterized by constant transformation.

The desire to maintain and continue the initial effectiveness of antibacterial therapy exceeded the real assessment of the new conditions and the impossibility of returning to the original state. This desire was logical, since at first it seemed that AP could only be treated with antibiotics without much effort. The further dynamics of the "microbe-antibiotic" ratio has long shown the deceptiveness of such expectations, but the subsequent transformation of views on the nature of this disease, which defies logical explanation, has further complicated the solution of the whole problem. The paradox is that the value and importance of antibiotics grew in parallel with the decline in their effectiveness and the increase in the number of resistant strains of microorganisms. Eventually, under the influence of the idea that AP should only be treated with antibacterial agents, the disease became classified as infectious.

The mental perception of the pathogen as the main cause of AP led to the oblivion of the fact that the disease is based on a non-specific inflammatory process and lung damage is accompanied by an inevitable violation of the unique functions of the organ. For the same reason, the growing need for additional means of assistance was compensated by the automatic application of techniques and methods that have proven themselves well in inflammatory processes with localization in the large circle of blood circulation.

In addition to these paradoxes and contradictions, at least in the last couple of decades, some experts began to pay attention to the increase in the number of observations of viral pneumonia, but such studies did not go beyond the statement of fact and statistics (4-6). The two major coronavirus epidemics, SARS and MERS, in the recent past also did not lead to radical changes in the concept and treatment of lung inflammation $(7,8)$. As a result, the SARS-CoV-2 pandemic clearly demonstrated the unpreparedness of modern medicine for a massive change in the etiology of lung inflammation and made the problem of AP visible and tangible to the widest audience.

Today, it is no secret that the main cause of morbidity and mortality in a pandemic is COVID-19 pneumonia. The change in etiology was accompanied by epidemiological, clinical and pathoanatomical nuances, but the essence of the disease remained the same and we continue to talk about the nosology of AP. Moreover, analysts note the great difficulty of differential diagnosis of this form of lesion from typical bacterial pneumonia $(9,10)$, despite the fact that both variants of inflammation have the same lethality (10).

In recent years, antibiotics continue to be considered a lifesaver for many people with AP. The sudden growth of viruses in the etiology of this disease showed that patients left without the main etiotropic treatment continue the natural division according to the severity of the disease in the same proportions that were observed in bacterial forms of inflammation against the background of "reasonable treatment". Despite the alarming public sentiment about the current pandemic, the very fact of infection with the coronavirus is not a fatal prognosis for the vast majority of its recipients. Up to $80 \%$ or more of infected people tolerate such contact without special medical care (11-15). Moreover, these statistics relate to the period before the start of vaccination of the population.

Even more impressive is the comparative mortality statistics. The most severe patients with AP are concentrated in intensive care units (ICU), where the majority of deaths are recorded. Currently, when the material on monitoring and supporting care (rather than treatment) for patients with COVID-19 pneumonia is accumulated, the results show that the mortality rates in this group do not differ from this indicator for bacterial forms of pneumonia - up to $30-50 \%$ (16-24). The difference is that with bacterial inflammation, everyone was sure that the patients received adequate care. Now there is a reasonable question about how adequate was the previous treatment, if up to half of these patients in the intensive care unit did not cope with the diseases, and now patients with COVID-19 show the same level of resistance, without having similar treatment? 
Such questions are quite logical, since the situation in the field of medical care for patients with AP requires a deep objective analysis and a radical revision of existing trends in solving this problem. Until now, the priority of the research and research work is focused on the dominant role of the pathogen in the features of the development of the disease. The chain of such dependence is clearly traced at different stages of the development of AP, both before and at the present time.

The presence of coronavirus in the body means only the fact of infection, which does not always lead to the disease. At the same time, the infection is not accompanied by external signs and special tests are needed to establish it. The development of the inflammatory process in the lung tissue means the emergence of new circumstances, which, first of all, will depend on the volume of the lesion. Hardly anyone will disagree with the opinion that $50 \%$ of lung tissue damage is accompanied by a more severe condition of the patient, compared to 5-10\%, right? However, the cause of the severity of the disease is considered to be its causative agent, and the inability to provide targeted assistance is due to the lack of antiviral drugs. A paradox? Undoubtedly, if we also take into account the fact that even in the presence of such drugs, they must penetrate into the affected cells and destroy pathogenic microorganisms, without directly affecting the restoration of impaired lung function.

With this variant of the main treatment, the body does not have time to adapt to the violation of its vital functions, especially with the aggressive development of the process. Such examples of the use of antibiotics in recent years have become quite frequent, when the process has reached the stage of complications and critical condition of the patient, despite the successful antibacterial treatment and the absence of microflora in the focus of inflammation.

Further progression of the disease leads to the development of septic shock. The very name of this severe complication indicates its source, but the genesis of shock in bacterial forms of AP in most patients was declarative and conjectural. Its septic origin was confirmed by bacteriological blood tests in isolated cases, not differing in the frequency of this test from similar patients without a shock reaction (2527). Similarly, septic shock continues to be interpreted at present as a consequence of viral overaggression, but again without objective evidence (28). And again, the essence of the problem is reduced to the causative agent of the disease, and the features of its pathogenesis remain outside the topic of discussion.

Thus, the problem of AP and the features of its manifestation at all stages of the disease are explained by the qualities of the pathogen, which are usually studied in vitro. The idea of what happens in the patient's body during the disease, as a rule, is created on the principle of analogies and assumptions. For example, a violation of gas exchange in the affected areas of the lungs is considered to be the cause of shortness of breath and hypoxemia, which seems to be a logical consequence of inflammatory tissue edema (29-31). But try to find an explanation for why a small focus of acute inflammation is characterized by more pronounced disorders than atelectasis, when the lobe or even the lung is disconnected from gas exchange, and you will not find modern interpretations of this difference.

A violation of gas exchange in the inflamed parts of the lungs suggests an improvement in this function due to the supply of oxygen and subsequent ventilation of the lungs. In this regard, ensuring the need for artificial lung ventilation continues to be considered as an important step in solving this problem (32-34). But, contrary to expectations, the results of such respiratory support only raise new questions. Thus, simple oxygen insufflation does not affect the course of the disease, and the use of artificial lung ventilation is a forced measure of support in the final stages and its use is naturally accompanied by a higher mortality rate $(12,22,24)$.

Similar, even more impressive discrepancies between the existing ideas and the actual data can be found in the analysis of circulatory disorders in patients with AP. The dominant ideas about circulatory disorders in patients with AP are in contradiction with the fundamental research on the role of the lungs in the vital activity of this system. The influence of the focus of inflammation on this function of the organ is replaced by the concept of the dependence of these disorders on the action of the infectious factor $(30,31,35-39)$. Such concepts suggest the wrong direction of therapeutic efforts, which may not bring the expected effect.

Antibacterial efforts, regardless of the location of the main focus of inflammation, combined with a gradual decrease in the effectiveness of 
antibiotics are at the root of the causes of the long-term deepening of the AP problem. If an inflamed lung is treated in the same way as an inflamed intestine, an inflamed throat, an inflamed eye, etc., can we expect stable successful results of such treatment, especially in conditions of rapid development of the process? The current results of AP treatment fully correspond to the narrowed ideas about the nature of the disease and the principles of medical care. From this point of view, a further increase in the frequency of pleural empyema in the last decade is quite natural, especially since $\mathrm{AP}$, as the main cause of this purulent complication, remains without a detailed analysis $(40,41)$.

Now comes the viral season in the etiology of AP, but the search for optimal treatment options continues on the basis of previous conceptual views on this disease. The purpose of such studies is to study the features of COVID-19 pneumonia based on the properties of the coronavirus and the expected consequences of its aggression $(30,31,35-39$,). At the same time, inflammation of the lung tissue, which is a distinctive characteristic of the disease and determines the originality and severity of its course, is considered as a sign, and not its basis. Various disorders that appear only from the moment of the development of the focus of inflammation are subjected to attempts to neutralize as the consequences of infection, and not damage to the functions of the organ (42-44).

The dominant perception of AP as a process that depends on its pathogen actually replaces the pathogenesis of the disease. Many mechanisms of disease development are often explained by the properties of the pathogen and its features. The study of the pathogenesis of AP is focused on the cellular-molecular level, where the reasons for the aggressiveness of pathogens and possible ways of protection are clarified. The effect of the inflammatory zone in the lung on the patient's body is not directly evaluated. Positive and encouraging results of such examinations, which are the hope for patients, always remain in the waiting mode.

The validity of the research goals determines their final results. Today's results show that the solution to the problem, despite a lot of work in this direction, did not lead to the achievement of the goal. In the 10 months of last year, since the announcement of the SARS-CoV-2 pandemic, 4,000 studies of the infection were registered worldwide, of which 1,500 were related to the development of drugs and vaccines (45). The issues of financial support for the research that this publication focuses on are extremely important, but the return on investment in the form of results achieved is equally important. Direct counteraction to the pathogen was successful only in the development of vaccines, but drug care during the disease period did not move from the previous positions.

Research on this issue is currently receiving generous support, but the lack of return on investment raises new questions. For example, by January 2021, the National Institutes of Health (US) had issued almost a thousand awards totaling about $\$ 2$ billion to support COVID-19 projects. However, the results of such studies remain largely unknown, and only $8 \%$ of completed or discontinued studies are published (46). But the main conclusion is that these incentives have not brought any improvements in the treatment of patients with COVID-19 pneumonia.

Summing up the general situation in solving the problem of AP, we can clearly note the tendentiousness and subjectivity of the prevailing ideas about the nature of the disease today. The long-standing cult of antibiotics gradually formed an abstract picture of the disease, in which everything depends on the microbiological agent, and the decisive role of inflammation as the main pathological process was no longer perceived in a positive way. The existing belief in the complete dependence of the development and course of AP on its causative agent does not agree and even contradicts many classical provisions of medical science. The causal chain of AP pathogenesis was replaced by a simple concept of direct action of the pathogen. Such an ideology of the problem, which determines the goals of its research and solution, does not even allow us to predict the results of the strategic level.

Thus, the inevitable step, without which a successful solution to the problem of AP is unthinkable, is to bring the ideology of this disease in line with the fundamental provisions of medical science. The so-called psychoanalysis of the modern perception of the problem of AP allows us to note the causes and main defects of the current concept. An additional incentive for complex psychological adaptation is the results of the work already done on the basis of the new teaching about the disease (47). The results of this work are the best example and a convincing argument for all of the above. 


\section{REFERENCES}

[1] Psychoanalysis.- https://en.wikipedia. org/wiki /Psychoanalysis

[2] Feigin R (2004). Textbook of Pediatric Infectious Diseases (5th ed.). Philadelphia: W. B. Saunders. p. 299. ISBN 978-0-7216-9329-3.

[3] Plotkin, Stanley; Orenstein, W; Offit, PA (September 22, 2012). Vaccines. Elsevier Saunders. p. 542. ISBN 978-1455700905. Retrieved July 2, 2015.

[4] Rudan I, Boschi-Pinto C, Biloglav Z, Mulholland K, Campbell H. Epidemiology and etiology of childhood pneumonia. Bull World Health Organ. 2008; 86:408-416. [PMC free article] [PubMed] [Google Scholar]

[5] WHO Revised global burden of disease 2002 estimates. 2004. http://www.who.int/ healthinfo/ global_burden_disease/estimates_ regional_2002_revised/en/ (accessed Nov 5, 2010).

[6] Ruuskanen O, Lahti E, Jennings LC, Murdoch DR (April 2011). "Viral pneumonia". Lancet. 377 (9773): 1264-75. doi:10.1016/S01406736(10)61459-6.

[7] SARS.https://en.wikipedia.org/wiki/Severe_acu te_respiratory_syndrome

[8] MERS.https://en.wikipedia.org/wiki/Middle_Ea st_respiratory_syndrome

[9] C. Heneghan, A. Pluddemann, K. R. Mahtani (2020). Differentiating viral from bacterial pneumonia. April 8, 2020. The Centre for Evidence-Based Medicine. Evidence Service to support the COVID-19 response. University of Oxford. https://www.cebm.net/covid-19/ differentiating-viral-from-bacterial-pneumonia.

[10] Kamat IS, Ramachandran V, Eswaran H, Guffey D, Musher DM. (2020). Procalcitonin to Distinguish Viral From Bacterial Pneumonia: A Systematic Review and Meta-analysis. Clin Infect Dis. 2020 Jan 16;70(3):538-542. doi: 10.1093/cid/ciz545. PMID: 31241140.

[11] Z. Wu, J. M. McGoogan (2020). Characteristics of and Important Lessons From the Coronavirus Disease 2019 (COVID-19) Outbreak in China. Summary of a Report of 72314 Cases From the Chinese Center for Disease Control and Prevention. JAMA. 2020; 323(13):12391242.doi:10.1001/jama.2020.26 48

[12] Merad, M., Martin, J.C.(2020). Pathological inflammation in patients with COVID-19: a key role for monocytes and macrophages. Nat Rev Immunol 20, 355-362 (2020). https://doi.org/ 10.1038/s41577-020-0331-4

[13] Ing AJ, Cocks C, Green JP (2020). COVID-19: in the footsteps of Ernest Shackleton. Thorax, 2020;75:693-694.

[14] Keeley AJ, Evans CM, de Silva TI (2020). Asymptomatic SARS-CoV-2 infection: the tip or the iceberg? Thorax 2020;75:621-622.
[15] A.Sakurai, T. Sasaki, S. Kato et al. (2020). Natural History of Asymptomatic SARS-CoV2 Infection. NEJM, June 12, 2020, DOI: 10.1056/NEJMc2013020

[16] A. Liapikou, E. Rosales-Mayor, A. Torres (2014). The management of severe community acquired pneumonia in the ICU. June 2014, Expert Review of Respiratory Medicine 8(3): 293-303.DOI: 10.1586/17476348.2014.896202

[17] J. W. Kim, , J.J. Kim, H. J. Yang at al.(2015). The Prognostic Factors of Pneumonia with Septic Shock in Patients Presenting to the Emergency Department. Korean Journal of Critical Care Medicine 2015; 30(4): 258-264

[18] P. Jason, N. C. Dean, Q. Guo et al. (2016). Severe community-acquired pneumonia: timely management measures in the first 24 hours. Critical Care,2016,20:237

[19] A. Vidal, L. Santos (2017). Comorbidities impact on the prognosis of severe acute community-acquired pneumonia. Porto Biomedical Journal, Vol. 2. Num. 6. November - December 2017, pages 247-346

[20] A. Ceccato, A. Torres (2018). Sepsis and community-acquired pneumonia. Ann Res Hosp 2018; 2:7

[21] Grasselli G et al. (2020). Baseline characteristics and outcomes of 1591 patients infected with SARS-CoV-2 admitted to ICUs of the Lombardy region, Italy. JAMA $2020 \mathrm{Apr}$ 6; [e-pub]. (https://doi.org/10. 1001/jama. 20 20.5394)

[22] S. Richardson, J. S. Hirsch, M. Narasimhan at al. (2020). Presenting Characteristics, Comorbidities, and Outcomes Among 5700 Patients Hospitalized With COVID-19 in the New York City Area. JAMA. Published online April 22, 2020. doi:10.1001/jama.2020.6775

[23] Renato Seligman and Beatriz Graeff Santos Seligman (2020). "Pandemic in the 21st Century. The Challenge of COVID-19". EC Pulmonology and Respiratory Medicine 9.8 (2020): 30-31.

[24] Gupta S, Wang W, Hayek SS, et al. Association between early treatment with tocilizumab and mortality among critically ill patients with COVID-19. JAMA Intern Med. 2020; 180 (11):1436-1446.doi:10.1001/jamainternmed. 2020.3596

[25] AJ Morgan and AJ Glossop. "Severe community-acquired pneumonia". BJA Education.2016, 16.5: 167-172.

[26] Garcia-Vidal C, Ardanuy C, Tubau F, et al. (2010). Pneumococcal pneumonia presenting with septic shock: host- and pathogen-related factors and outcomes. Thorax 2010;65:77-81.

[27] Hadil A.Al Otair, Mohammed A.Hussein, Mohamed A.Elhoseny et al. (2015). Severe pneumonia requiring ICU admission: Revisited. 
Journal of Taibah University Medical Sciences. Volume 10, Issue 3, September 2015, Pages 293-299. https://doi.org/10.1016/ j.jtumed. 20 15.03.005

[28] Alhazzani W et al. (2020). Surviving sepsis campaign: Guidelines on the management of critically ill adults with coronavirus disease 2019 (COVID-19). Crit Care Med 2020 Mar 27; [e-pub]. (https://doi.org/10. 1097/CCM. 0000000000004363)

[29] Pfeifer M, Hamer OW. (2020). COVID-19Pneumonie [COVID-19 pneumonia]. Internist (Berl). 2020 Aug; 61(8):793-803. German. doi: 10.1007/s00108-020-00854-5. PMID:3272881 7 ; PMCID: PMC7388437.

[30] Attaway A H, Scheraga R G, Bhimraj A et al. (2021). Severe covid-19 pneumonia: pathogenesis and clinical management. BMJ 2021; 372 :n436 doi:10.1136/bmj.n436

[31] M. K. Bohn, A. Hall, L. Sepiashvili et al. (2020). Pathophysiology of COVID-19: Mechanisms Underlying Disease Severity and Progression. Physiology 2020 35:5, 288-301

[32] J. Gafford et al. (2021). "The Vanderbilt OpenSource Ventilator: From Napkin Sketch to Ready to Save Lives in Three Weeks," in IEEE Robotics \& Automation Magazine, vol. 28, no. 1, pp. 101-114, March 2021, doi: 10.1109/ MRA.2020.3045668.

[33] Rice TW, Janz DR. (2020). In Defense of Evidence-based Medicine for the Treatment of COVID-19 Acute Respiratory Distress Syndrome. Ann Am Thorac Soc. 2020 Jul; 17(7):787-789. doi: 10.1513/ AnnalsATS. 202004-325IP. PMID: 32320268; PMCID: PMC7328187.

[34] Truog RD, Mitchell C, Daley GQ. (2020). The toughest triage - allocating ventilators in a pandemic. N Engl J Med 2020;382:1973-1975.

[35] Loo J, Spittle DA, Newnham M. (2021). COVID-19, immunothrombosis and venous thromboembolism: biological mechanisms. Thorax 2021; 76:412-420

[36] Lipman M, Chambers RC, Singer M, et al. (2020). SARS-CoV-2 pandemic: clinical picture of COVID-19 and implications for research. Thorax 2020; 75:614-616.

[37] Mo Shehata (2020). "Covid-19; The Possible Medical Strategies". EC Pulmonology and Respiratory Medicine 9.6 (2020): 03-07.
[38] El-Shimy, IA, Mohamed, MM, Hasan, SS, Hadi, MA. (2021). Targeting host cell proteases as a potential treatment strategy to limit the spread of SARS-CoV-2 in the respiratory tract. Pharmacol Res Perspect. 2021; 9:e00698. https://doi.org/10.1002/prp2.698

[39] V. J. Dzau, C. Balatbat (2020). Strategy, coordinated implementation, and sustainable financing needed for COVID-19 innovations. The Lancet Journal, Nov 07, 2020, Volume 396, Number 10261p1463-1534, e73-e82. DOI: https://doi.org/10.1016/S0140-6736(20)32289-3

[40] Arnold D, Hamilton F, Morris T, et al. (2019). The changes in incidence and management of pleural empyema in England over the last decade. Thorax, 2019;74:A9-A10.

[41] Bobbio A, Bouam S, Frenkiel J, et al. (2021). Epidemiology and prognostic factors of pleural empyema. Thorax, Published Online First: 30 March2021.doi:10.1136/thoraxjnl-2020-215267

[42] Farah H, et al.(2020). Potential AntiInflammatory Approaches for the Management of SARS-CoV2 Infections. J Pulmon Respir Sci 2020, 5(S1): 000S1-001.

[43] Ahmed I. Siddiqi., (2020) Cytokine flames of COVID-19. J. Archives of Medical Case Reports and Case Study. 3(2); DOI:10. 31579/2692-9392/014

[44] Christiansen CF, et al. (2020). SARS-CoV-2 infection and adverse outcomes in users of ACE inhibitors and angiotensin-receptor blockers: a nationwide case-control and cohort analysis. Thorax, 2020;0:1-10. doi:10.1136/ thoraxjnl-2020-215768

[45] K. Armstrong (2021). Covid-19 and the Investigator Pipeline. March 24, 2021. DOI: 10.1056/NEJMp2100086

[46] K. T. Kadakia, A. L. Beckman, J. S. Ross et al. (2021). Leveraging Open Science to Accelerate Research. March 24, 2021, DOI: 10.1056/ NEJMp20345

[47] Igor Klepikov (2020). Acute pneumonia. New doctrine and first treatment results. Lambert Academic Publishing, 2020. ISBN (978-620-267917-6)

Citation: Igor Klepikov, MD, "Psychoanalysis of the Doctrine of Acute Pneumonia”, International Journal of Research Studies in Medical and Health Sciences. 2021; 6(3): 21-26. DOI: https://doi.org/10.22259 /ijrsmhs.0603005

Copyright: () 2021 Igor Klepikov, MD, This is an open-access article distributed under the terms of the Creative Commons Attribution License, which permits unrestricted use, distribution, and reproduction in any medium, provided the original author and source are credited. 